\title{
Validación de una rúbrica para las competencias docentes en el marco de los aprendizajes clave
}

Validation of a rubric for teaching competencies in the framework of key learnings

\section{Florentino López}

Universidad Pedagógica Nacional - México

Chilpancingo, México

mercurius6o@hotmail.com

\section{Resumen}

Debido a las escasas investigaciones e instrumentos que valoren las competencias docentes en el marco de los aprendizajes clave, el artículo resume el diseño y validación de una rúbrica orientada a evaluar sus saberes pedagógicos en el campo educativo. Para el diseño del instrumento fueron considerados diez ítems con sus respectivos descriptores de desempeño relativos a evaluar las competencias docentes sobre los aprendizajes clave. Para el proceso de validación los ítems fueron revisados por tres expertos quienes aportaron sugerencias de sintaxis y redacción orientadas a mejorar la comprensión e interpretación. En la siguiente fase el instrumento fue evaluado por un equipo de trece jueces expertos, quienes aportaron una evaluación respecto a la validez de contenido de los ítems y descriptores de desempeño, obteniéndose valores óptimos por cada ítem ( $v$ de Aiken>0.80), tanto en pertinencia como en redacción, manifestando un excelente grado de comprensión de instrucciones e ítems, por lo que se considera que el instrumento es factible para valorar las competencias docentes referidas.

Palabras clave: Aprendizajes clave; competencias docentes; rúbrica analítica; validez; confiabilidad

\section{Abstract}

Considering the limited research and instruments that assess teaching competencies within the framework of key learning, the article summarizes the design and validation of a rubric aimed at assessing their pedagogical knowledge in the educational field. Ten items were considered for the design of the instrument with their respective performance descriptors related to the evaluation of teaching competencies on key learning. For the validation process, the items were reviewed by three experts who provided syntax and wording suggestions aimed at improving comprehension and interpretation. In the next phase, the instrument was evaluated by a team of thirteen expert judges, who provided an assessment of the content validity of the items and performance descriptors, obtaining optimal values for each item (Aiken's $v>0.80$ ), both in relevance and wording, showing an excellent degree of understanding of instructions and items, therefore, it is considered that the instrument is feasible to assess the teaching competencies..

Keywords: Key learnings; Teaching competences; analytical rubric; validity; reliability 


\section{Introducción}

Valorar las competencias docentes en el marco de los aprendizajes clave para la educación integral del nuevo modelo educativo, requiere de un análisis reflexivo, crítico y propositivo de los saberes pedagógicos y habilidades cognitivas que los educadores ponen en juego para conducir el proceso de aprendizaje con eficiencia y eficacia. Las competencias son saberes concatenados que integran el ser, el saber hacer y el saber estar; el domino de estos saberes conceptuales, procedimentales y actitudinales, determinan en buena medida la capacidad para actuar con efectividad frente a contextos escolares y laborales (Ríos 2017, Tobón 2013, Castañeda, 2018).

En este sentido, se requiere de herramientas para valorar las competencias docentes sobre los aprendizajes clave que se establecen en el nuevo modelo educativo, es decir, ¿Qué saben los educadores sobre el nuevo enfoque educativo? ¿Conocen la organización curricular de los aprendizajes clave? ¿Cuáles son sus competencias pedagógicas y didácticas para su abordaje en el aula? El ser competente refiere tener la capacidad de disponer de un bagaje educativo y los recursos necesarios para emprender y desarrollar una actividad de forma pertinente y eficaz (Tejada, 2016). Las competencias se construyen como la articulación de habilidades cognitivas y prácticas, como cimientos, motivaciones, valores, actitudes, emociones y de otros aspectos sociales y conductuales; residen en la capacidad de movillizar los saberes intelectuales y contextuales para resolver problemas, aplicando la reflexión, acción y transferencia orientadas a la mejora de la situación entrentada (Lima, 2015).

El modelo de competencias exige que los docentes motiven a los alumnos, organicen ambientes y experiencias de aprendizaje que los guíen en la construcción de su propio conocimiento, considerando para ello emplear las metodologías, las estrategias y los recursos que faciliten a los estudiantes la adquisición y desarrollo de competencias. En este contexto, Zabalza (2005), señana diez competencias que deben asumir el profesorado para operativizar el proceso de enseñanza-aprendizaje: capacidad de planificar el proceso de educativo, seleccinar y presentar los contenidos de aprendizaje, ofrecer información y explicaciones comprensibles, alfabetización tecnológica y manejo didáctico de las TIC, gestionar las metodologías de trabajo didáctico y las tareas de aprendizaje, relacionarse constructivamente con los alumnos, brindar acompañamiento a los estudiantes, reflexionar e investigar sobre la práctica e implicarse institucionalmente.

Atendiendo lo anterior, se deriva la importancia de valorar las competencias docentes sobre el nuevo modelo educativo, indagar qué conocimientos poseen sobre los aprendizajes clave, cuál es la metodología de enseñanza que se plantea, la organización curricular de los aprendizajes esperados, el papel que juegan los educadores en ese nuevo paradigma de la enseñanza , las formas de planificación, de abordaje didáctico y los procesos de evaluación que se describen en los enfoques de cada asignatura. La valoración de las competencias docentes como procesos complejos que las personas ponen en acción-actuación-creación (Tobón, 2005), para desempeñar actividades en el ámbito profesional, puede brindar información relevante para promover un análisis y reflexión sobre la práctica educativa, identificar retos y desafíos del profesorado ante la demanda de las generaciones del pleno siglo XXI.

En este ámbito, se encontraron escasos instrumentos que tengan por objetivo valorar las competencias docentes en el marco de los aprendizajes clave; al respecto, se encontró un 
artículo de Tobón et al., (2018) denominado: "Validez y confiabilidad de una rúbrica para evaluar las paracticas pedagógicas en docentes de educación media (socme-10)" en el cual se describe el proceso de validación y confiabilidad del instrumento compuesto por diez ítems orientados a evaluar diez prácticas pedagógicas relativas a los procesos de motivación, aprendizaje de conceptos, resolución de problemas, proyecto ético de vida, comunicación asertiva, trabajo colaborativo, desarrollo de la creatividad, transversalidad, gestión de recursos y evaluación formativa; éstas, centradas en la autoevaluación del desempeño docente en el aula.

Por otra parte, Arévalo et al., (2020) confirman que el uso de la rúbrica como herramienta de evaluación, permite identificar los logros alcanzados por los maestros en los procesos pedagógicos que desarrollan en las aulas; Cano (2015), hace unanálisis argumentativo sobre las ventajas y desventajas del del uso de la rúbrica para evaluar comportamientos observables y concluye que estos instrumentos son de gran utilidad para autorregular el aprendizaje, siempre y cuando sean acompañadas con procesos de formación y estudios de validez y fiabilidad de contenido; Hernández y Cerda (2019), diseñaron una rúbrica para evaluar la planificación didáctica a partir de dos dimensiones y ocho indicadores que tienen por objetivo valorar el nivel de dominio con el que se realiza el diagnóstico del grupo, los elementos curriculares, el abordaje de problemas del entorno, la secuenciación de actividades, los recursos didáctivos, la oganización del grupo y la evaluación del aprendizaje, como componentes centrales de la planificación didáctica, pero no se valoran las competencias docentes en su conjunto en el campo de actuación.

Por lo anterior, el presente artículo tiene como objetivo validar una rúbrica analítica para valorar las competencias docentes en el marco de los aprendizajes clave. Hacer un análisis de las competencias pedagógicas del profesorado frente a los aprendizajes clave que se definen en los programas de estudio del nuevo modelo educativo para la educación obligatoria, puede brindar ciertos referentes de los conocimientos, habilidades, destrezas, estrategias y metodologías didácticas con los cuales los maestros enfrentan los nuevos desafíos que demanda la reforma educativa en los procesos de enseñanza-aprendizaje, con el propósito de generar espacios de autorreflexión de la práctica pedagógica y diseñar acciones de capacitación y actualización profesional.

El estudio está orientado a lograr las siguientes metas: 1) establecer la validez de contenido de la rúbrica en docentes de educación primaria mediante la evaluación de un grupo de jueces expertos; 2) Identificar el grado de pertinencia y redacción del instrumento; 3) analizar la confiabilidad del instrumento mediante la aplicación a un grupo piloto para valorar el grado de satisfacción y de consistencia interna a partir del coeficiente de Alfa de Cronbach.

\section{Metodología}

La presente investigación es de tipo instrumental de conformidad con la clasificación que establece Montero y León (2002), en cuanto estuvo enfocada al diseño de la rúbrica analítica como instrumento que tiene el propósito de evaluar las competencias de los maestros en el marco de los aprendizajes clave; considerando que las investigaciones de tipo instrumental tienen como objetivo proponer la creación de un instrumento de evaluación para aplicarlo en los nuevos contextos educativos y valorar su pertinencia y confiabilidad (Argumedo, 2016). En esta 
perspectiva, se describen a continuación las fases del proceso metodológico que se llevó a cabo para el diseño, pertinencia, validez y confiabilidad del instrumento de investigación para lograr su consistencia y relevancia.

\subsection{Diseño y revisión por expertos}

Se diseñó una rúbrica analítica de tipo socioformativa como instrumento de evaluación para valorar las competencias de los educadores con respecto a los aprendizajes clave como elementos esenciales del currículo que se proponen en el nuevo modelo educativo para la educación obligatoria (Secretaría de educación pública, 2017). El instrumento está compuesto por 10 ítems, cada ítem contiene cinco descriptores que gradúan el nivel de competencia del docente con referencia a cada indicador que se relaciona con los aprendizajes clave o el constructo a evaluar.

Los niveles y descriptores de desempeño tienen la intencionalidad de promover en los docentes la reflexión sobre sus competencias e incidir de forma sugestiva procesos de realimentación y actualización pedagógica. Los niveles de desempeño con su respectivo descriptor se graduaron en orden ascendente para indicar el grado de competencia que refiere el docente al ser valorado, estableciéndose los niveles de la siguiente forma: nivel preformal (muy bajo), nivel receptivo (bajo), nivel resolutivo (medio), nivel autónomo (medio alto) y nivel estratégico (muy alto) (Anexo 1).

En la primera fase, la rúbrica analítica fue revisada por tres expertos en diseño y evaluación de instrumentos de investigación, con el objeto de hacer una revisión de los ítems y descriptores de cada nivel de desempeño y aportar sugerencias y recomendaciones enfocadas a dar mayor calidad a la sintaxis y redacción de las preguntas. Como resultado del proceso de validación facial por parte de los tres expertos quienes, de acuerdo a su perfil profesional y conocimiento sobre el tema, consideraron los criterios de gramática, cohesión y coherencia en la redacción de los indicadores y descriptores de la rúbrica (Parrado, 2016). Para su caracterización se empleó el cuestionario de factores sociodemográficos creado por CIFE, (2015), el cual tiene el propósito de recabar información sobre el nivel académico de los participantes, campo donde desempeñan su función docente y años de experiencia laboral en el ramo y experiencia en la revisión y diseño de instrumentos de investigación, así como otros datos relacionados con el ámbito profesional (tabla 1).

Tabla 1. Datos sociodemográficos de los expertos

\begin{tabular}{ll}
\hline Sexo & $\begin{array}{l}33.3 \% \text { hombres } \\
66.6 \% \text { mujeres }\end{array}$ \\
\hline Roles & Docentes: 3 \\
& Directivos: 0 \\
Último grado de estudios & Maestría: 2 \\
& Doctorado: 1 \\
Área de experiencia profesional: & Docencia e investigación. \\
Número de años de experiencia profesional: & 24 \\
Número de artículos publicados en el área: & 4 \\
Número de ponencias presentadas en el área: & 7 \\
Número de libros publicados en el área: & 2 \\
Experiencia en la revisión, diseño y/o validación en & \\
un determinado instrumento de investigación: & Sí \\
\hline
\end{tabular}

Fuente: elaborado por el autor. 


\subsection{Juicio de expertos}

Para el análisis de la validez de contenido del instrumento, se realizó un juicio de expertos, en el cual participaron 13 jueces expertos (ver tabla 2), quienes de acuerdo a su experiencia y conocimiento sobre el tema a evaluar, formación académica y experiencia en revisión y diseño de instrumento de investigación, son reconocidos por otros como expertos cualificados para aportar su valoración u opinión informada (Escobar, 2008; García, 2018; Juárez-Hernández y Tobón, 2018) sobre el grado de pertinencia y redacción de los indicadores y descriptores que conforman la rúbrica analítica, respectivamente.

Se realizó un análisis con enfoque cualitativo y cuantitativo de cada uno de los ítems para la evaluación de la pertinencia y relevancia de los mismos, valorando los indicadores y descriptores de los niveles de desempeño a partir de la "escala de jueces" (CIFE, 2018a). En este proceso y de conformidad con su experiencia y formación académica respectiva, los jueces expertos pueden aportar observaciones y sugerencias que consideren pertinente para la mejora en la redacción de cada ítem, indicadores y descriptores de desempeño del instrumento para que sea comprensible y de calidad.

Para valorar la pertinencia y redacción de los indicadores, se utilizó una escala tipo Likert con valores de 1 a 4 (Escala de jueces expertos). El análisis de la evaluación cuantitativa se realizó por medio de la $\mathrm{V}$ de Aiken para cuantificar el acuerdo entre jueces, considerando que el valor de $\mathrm{V}$ puede oscilar entre cero y la unidad, y que las evaluaciones que están más próximas a la unidad, son las que describen la mayor magnitud posible que indican un perfecto acuerdo entre los jueces respecto a la validez de contenido de los ítems y los descriptores evaluados del instrumento de investigación, considerando que el estándar mínimo más exigente es 0.70 (Merino, 2009; JuárezHernández y Tobón, 2018). Los ítems que reflejaran observaciones o sugerencias, serían revisados en su redacción y estructura sintáctica para mejorar la coherencia y comprensión de los mismos.

Los criterios que se utilizaron para evaluar la pertinencia de cada ítem fueron: 1) No es pertinente. El ítem no contribuye a evaluar el propósito y dimensiones del instrumento en ningún aspecto, 2) Bajo nivel de pertinencia. El ítem hace una contribución superficial a la evaluación del propósito y dimensiones del instrumento, 3) Aceptable grado de pertinencia. El ítem contribuye a evaluar el propósito, dimensiones y/o constructo teórico del instrumento, 4) Alto nivel de pertinencia. El ítem contribuye a evaluar en un alto grado el propósito, dimensiones y/o constructo del instrumento.

Con relación a la evaluación de la redacción de cada ítem, los criterios fueron los siguientes: 1) No es comprensible. El ítem resulta escasamente comprensible para los usuarios del instrumento, 2) Bajo nivel de comprensión. El ítem debe ser mejorado en al menos la mitad de sus componentes, en aspectos de redacción y gramática, 3) Aceptable nivel de comprensión. El ítem requiere algunas mejoras superficiales para que quede excelente en su comprensión, y 4) Alto nivel de comprensión. El ítem es sumamente comprensible por los potenciales usuarios y sigue las normas gramaticales de la lengua. La ponderación de los criterios anteriores, definieron la pertinencia y redacción de cada uno de los ítems. 
Tabla 2. Datos de las competencias de los jueces.

\begin{tabular}{ll}
\hline Sexo & $\begin{array}{l}46.15 \% \text { hombres } \\
53.85 \% \text { mujeres }\end{array}$ \\
\hline Roles & $\begin{array}{l}\text { Docentes: } 100 \% \\
\text { Directivos: } \% \%\end{array}$ \\
Último grado de estudios & Maestría: $38.4 \%$ \\
& Doctorado: $61.5 \%$ \\
& Posdoctorado: $0 \%$ \\
Área de experiencia profesional: & Docencia e investigación. \\
Número de años de experiencia profesional: & 22.6 \\
Número de artículos publicados en el área: & 23 \\
Número de ponencias presentadas en el área: & 18 \\
Número de libros publicados en el área: & - \\
$\begin{array}{l}\text { Experiencia en la revisión, diseño y/o validación en un } \\
\text { determinado instrumento de investigación: }\end{array}$ & Sí
\end{tabular}

Fuente: elaborado por el autor.

\subsection{Aplicación de la prueba a un grupo piloto}

Con el propósito de obtener información relevante y sugerente para valorar el grado de satisfacción, calidad del instrumento y en su defecto mejorarlo de tal manera que sea pertinente, (Gómez, 2018) se aplicó la rúbrica analítica a un grupo piloto compuesto por diez docentes frente a grupo en educación primaria. Se incluyeron las preguntas sociodemográficas de los docentes participantes (ver tabla 3), los indicadores de la rúbrica de evaluación, las preguntas que abordan los ejes de grado de comprensión de las instrucciones, grado de comprensión de las preguntas o ítems, grado de satisfacción del instrumento y el grado de relevancia de las preguntas de la rúbrica analítica, mediante la aplicación de la encuesta de satisfacción del constructo a evaluar (CIFE, 2018b).

Para valorar los aspectos anteriores, se integró una escala numérica comprendida del 1 al 4, donde 1 indicaba bajo grado de aceptación y 4 excelente grado. Con los resultados de la valoración del grupo piloto, se realizó un análisis de la consistencia interna mediante el Alfa de Cronbach para evaluar la correlación de los ítems del instrumento (Pacheco, 2015). Las ponderaciones del grupo piloto, permite estimar la relevancia de las preguntas y la confiabilidad del instrumento de investigación.

Tabla 3. Datos sociodemográficos del grupo piloto.

\begin{tabular}{ll}
\hline \multicolumn{1}{c}{ Características } & Datos \\
\hline Sexo & $\begin{array}{l}40 \% \text { hombres } \\
60 \% \text { mujeres }\end{array}$ \\
Roles & Docentes: 10 \\
Último grado de estudios & Licenciatura: 5 \\
& Maestría: 5 \\
Promedio de edad en años: & 33.7 \\
Zona de residencia: & Urbana \\
Número de años de experiencia laboral: & 10.6 \\
Experiencia en la revisión, diseño y/o validación en un de- & \\
terminado instrumento de investigación: & Sí \\
Condiciones económicas: & $100 \%$ Media \\
\hline
\end{tabular}

Fuente: elaborado por el autor. 
Aspectos éticos. La aplicación de la rúbrica analítica para valorar las competencias docentes en el marco de los aprendizajes clave, estuvo enmarcada en la observancia de los aspectos éticos que constituye un elemento central al momento de iniciar y desarrollar cualquier estudio investigativo (Moscoso, 2018). En este ámbito, y con el propósito de asegurar la vinculación voluntaria de los destinatarios, se hizo la invitación respetuosa a los participantes, explicando la naturaleza y el objetivo de la investigación, la confidencialidad de la información y la no implicación de los resultados en algún aspecto de su vida personal o profesional.

\section{Resultados}

En la primera fase de revisión por tres expertos, se obtuvieron observaciones y sugerenccias relativas a la gramática y sintaxis de dos ítems y descriptores de desempeño, específicamente en el empleo de los verbos y la estructura sintáctica, las cuales se atendieron y se realizaron las modificaciones y adecuaciones pertinentes a cada uno de ellos con el propósito de concluir la redacción final de los ítems y descriptores del instrumento para someterlo a la siguiente etapa de validez de contenido por un grupo de jueces expertos.

Los resultados de las evaluaciones y ponderaciones que realizó el grupo de jueces expertos de cada uno de los ítems y descriptores de desempeño del instrumento, fueron sometidos a análisis estadístico mediante el coeficiente de validez de contenido ( $V$ de Aiken), reflejando que las preguntas tienen un aceptable nivel de pertinencia y redacción, al observar que los valores obtenidos fueron mayor a 8.0, lo que demuestra la validez de la rúbrica (tabla 4).

Sin embargo, a pesar de que la evaluación cuantitativa de los jueces expertos fue validada con una puntuación aceptable, en la valoración cualitativa se obtuvieron sugerencias y observaciones para mejorar la redacción del instrumento con el fin de dar mayor claridad a la sintaxis y coherencia a los ítems 3, 4 y 9; así como los descriptores de cada nivel de desempeño. Las observaciones fueron orientadas a mejorar la redacción de los ítems descritos y la estructura sintáctica de los descriptores de cada nivel de desempeño con el objeto de no generar confusión ni sugerir tendencias para evitar sesgos de la investigación. En este sentido, las observaciones y recomendaciones planteadas, fueron atendidas a partir de la revisión y mejora de la redacción y estructura sintáctica de los ítems y descriptores señalados.

Tabla 4. Resultados del coeficiente de validez de contenido ( $V$ de Aiken) relativos a la pertinencia y redacción por ítem

\begin{tabular}{ccc}
\hline Item & Pertinencia & Redacción \\
1 & 0.923 & 0.923 \\
2 & 0.948 & 0.923 \\
3 & 0.923 & 0.871 \\
4 & 0.923 & 0.846 \\
5 & 0.948 & 0.923 \\
6 & 0.923 & 0.948 \\
7 & 0.974 & 0.948 \\
8 & 0.948 & 0.948 \\
9 & 0.897 & 0.897 \\
10 & 0.974 & 0.974 \\
\hline \multicolumn{3}{c}{ Fuente: Autor }
\end{tabular}




\subsection{Grupo piloto}

A partir de los resultados de esta última fase y con base en la encuesta que los docentes contestaron, se indicó un excelente grado de satisfacción con el instrumento en el área de la comprensión de las instrucciones de cada ítem, el grado de comprensión de las preguntas, así como el grado de relevancia de los ítems de la rúbrica analítica (Tabla 5), obteniéndose comentarios positivos sobre el instrumento para valorar las competencias docentes en el marco de los aprendizajes clave. Respecto a la evaluación de la confiabilidad, de conformidad con las ponderaciones de la encuesta que efectuó el grupo piloto, se obtuvo un valor satisfactorio de la rúbrica (Alfa de Cronbach: 0.87), superior a 0.70 que es considerado el rango mínimo para que los ítems de un instrumento de investigación muestren correlación entre sí y definan la consistencia interna (Bojórquez, 2013; Juárez-Hernández, 2018), lo que indica una aceptable consistencia interna de los ítems del instrumento.

Tabla 5. Resultados de la encuesta de satisfacción con el instrumento por el grupo piloto.

\begin{tabular}{lccc}
\hline \multicolumn{1}{c}{ Ítems } & $\begin{array}{c}\text { Bajo } \\
\text { grado }\end{array}$ & $\begin{array}{c}\text { Aceptable } \\
\text { grado }\end{array}$ & $\begin{array}{c}\text { Buen grado } \\
\text { Excelente } \\
\text { grado }\end{array}$ \\
\hline $\begin{array}{l}\text { 1. ¿Cuál fue el gado de comprensión de las } \\
\text { instrucciones del instrumento? }\end{array}$ & & $40 \%$ & $60 \%$ \\
$\begin{array}{l}\text { 2. ¿Cuál fue el grado de comprensión de las } \\
\text { preguntas o ítems? }\end{array}$ & $50 \%$ & $50 \%$ \\
$\begin{array}{l}\text { 3. ¿Cuál fue el grado de aceptación con el } \\
\text { instrumento? }\end{array}$ & & $60 \%$ & $40 \%$ \\
$\begin{array}{l}\text { 4. ¿Cuál fue el grado de relevancia de las pre- } \\
\text { guntas? }\end{array}$ & & & $100 \%$ \\
\hline
\end{tabular}

Fuente: autor

\section{Discusión}

La rúbrica para evaluar las competencias docentes en el marco de los aprendizajes clave, posee niveles adecuados de validez de contenido y confiabilidad, por lo que se considera pertinente para valorar los saberes pedagógicos y didácticos de los maestros frente a los procesos de enseñanza-aprendizaje que se gestionan en las aulas, puesto que considera aspectos centrales de la práctica docente y cumple con el criterio de desempeño que sirve para inferir el estado de competencia de una persona (García-Valcalver et al. 2020). Como señala Valverde (2014), las rúbricas son guías de puntuación que se utilizan en la valoración del desempeño de una persona y describen las características específicas de un producto, proyecto o tarea en varios niveles de rendimiento. En el caso que nos ocupa, la rúbrica evalúa las competencias que poseen los educadores para enfrentar la tarea de la enseñanza y generar ambientes de aprendizaje que respondan a las necesidades educativas y la sociedad del conocimiento.

Los resultados obtenidos mediante las ponderaciones emitidas por los evaluadores expresan una congruente concordancia entre las puntuaciones en el total de la rúbrica, lo que indican valores positivos que garantizan la fiabilidad del instrumento validado. El alcance de la rúbrica está enfocado a rescatar las competencias de los docentes sobre el dominio de los aspectos conceptuales y organización curricular de los aprendizajes clave, las estrategias de planificación didáctica, de enseñanza, de evaluación, de gestión del trabajo colaborativo, de competencias 
socioemocionales y de resolución de problemas del contexto que ponen en juego para favorecer la construcción social del conocimiento y el desarrollo de procesos metacogntivos en los estudiantes.

Diversos estudios se han centrado en el diseño de instrumentos para valorar los sabres docentes en el marco de la práctica pedagógica, Hernández y Cerda (2019), elaboraron una rúbrica para evaluar el diseño y ejecución de la paneación didáctica mediante dos dimensiones y ocho indicadores pero no valoran su transposición en el campo de gestión del proceso de enseñanzaaprendizaje; Salazar-Gómez, Tobón y Juárez-Hernández (2018), plantearon una rúbrica para evaluar las competencias digitales de los docnetes a través del diseño de site indicadores relacionados con la alfabetización informacional, comunicación y colaboración, creación de contenido digital, seguirdad, resolución de problemas, cuidado del medio ambiente y uso de las tic, pero al igual que el anterior, no se abordan en su conjunto las dimensiones que reflejan las prácticas educativas enel aula.

Por otra parte, Tobón et al. (2018) plantea una rúbrica para evaluar las prácticas pedagógicas en docentes de educación media, donde se describen diez prácticas relacionadas con los procesos de motivación, aprendizaje de conceptos, resolución de problemas del contexto, formación de valores, comunicación asertiva, trabajo colaborativo, creatividad e innovación, transversalidads, gestión de recursos para resolver problemas y el empleo de la evaluación para el mejoramiento continuo; la rúbrica está centrada en la evaluación de la práctica pedagógica y fue evaluada con aceptable nivel de confiabilidad, validez de contenido, pertinencia, redacción y comprensión de ítems e indicadores de desempeño. Los hallazgos que se describen en este estudio, tienen congruente relación con el instrumento diseñado para valorar las competencias docentes en el marco delos aprendizajes clave.

Sin embargo, en el estudio de validez de una rúbrica para las competencias docentes en el marco de los aprendizajes clave, se incluyen tres indicadores que no se retoman en el instrumento para evaluar las prácticas pedagógicas como son: 1) gestiona información sobre la conceptualización y organización curricular de los aprendizaje clave, 2) planifica actividades acorde con las necesidades educativas de los alumnos y, 3) favorece en los alumnos el desarrollo de competencias socioemocionales, estos indicadores forman parte crucial de las competencias docentes para garantizar prácticas educativas sustentadas en la planificación de las actividades académicas derivadas de los contenidos curriculares, atendiendo las necesidades de aprendizaje de los alumnos y el desarrollo de competencias socioemocionales para favorcer la formación integral de los estudiantes (SEP, 2017).

En cuanto al análisis de los ítems, niveles de domino y descriptores de desempeño de la rúbrica, se puede argumentar que fueron evaluados con una puntuación aceptable en pertinencia, redacción, relevancia y confiabilidad, lo que avala su factibilidad para ser aplicada a una muestra representativa que pueda brindar información relevante sobre las competencias de los educadores frente a los aprendizajes clave para la educación integral; considerando que el uso de la rúbrica como instrumento de evaluación, permite identificar los logros alcanzados por los maestros en los procesos pedagógicos que se gestionan en el aula (Arévalo, Castro y Leguía, 2020). 


\section{Conclusiones}

El instrumento que se presenta es una rúbrica analítica de enfoque socioformativo para valorar las competencias docentes en el marco de los aprendizajes clave que se establecen en el nuevo modelo educativo para la educación integral, el cual permitirá a los educadores evaluar sus competencias y definir procesos de actualización o retroalimentación de sus saberes para mejorar su práctica docente y en consecuencia los procesos de enseñanza-aprendizaje de conformidad con las demandas de la sociedad del conocimiento. Las etapas del proceso de revisión, validez y confiabilidad, demuestran que el instrumento es apto para aplicarlo a una población más amplia de maestros con el objetivo de obtener referencias de sus competencias en el ámbito de su práctica educativa.

Si bien, el instrumento de investigación pretende tener un acercamiento sobre los conocimientos de los maestros sobre los aprendizajes esperados que enmarca el nuevo currículo escolar, hace falta profundizar en las investigaciones para diagnosticar y empoderar a los educadores sobre los nuevos planteamientos curriculares y los desafíos que representa la sociedad del conocimiento. En este ámbito, se sugiere desarrollar nuevas investigaciones con mayor rigurosidad en validez de contenido y fiabilidad en diversos contextos para realizar contrastes y mejorar los hallazgos en torno a la rúbrica como instrumento de evaluación de las competencias docentes.

\section{Referencias}

Aravena, P.C., Moraga, J., Cartes-Velásquez, R., y Manterola, C. (2014).Validez y confiabilidad en investigación odontológica. International journal of odontostomatology, 8(1), 69-75. http://dx.doi.org/10.4067/So718$381 \times 2014000100009$

Arévalo, J.C., Castro, W.G., \& Leguía, Z.J. (2020). La rúbrica como instrumento de evaluación y el desempeño docente con enfoque intercultural en instituciones educativas de primaria en Perú. Revista Conrado, 16(73), 14-20. https://conrado.ucf.edu.cu/index.php/conrado/article/view/1264

Argumedo, D., Nóblega, M., Bárrig, P., y Otiniano, F. (2016). Criterios homologados de investigación en psicología (CHIP) investigaciones instrumentales versión 1.o. Departamento de Psicología PUCP

Bojórquez Molina, J. A., López Aranda, L., Hernández Flores, M. E., y Jiménez López, E. (2013). Utilización del alfa de Cronbach para validar la confiabilidad de un instrumento de medición de satisfacción del estudiante en el uso del software Minitab [Discurso principal]. 11th Latin American and Caribbean Conference for Engineering and Technology, Cancún, México. http://laccei.org/LACCEl2013-Cancun/ RefereedPapers/RP065.pdf

Cano, E. (2015). Las rúbricas como instrumento de evaluación de competencias en educación superior: ¿uso o abuso? Profesorado, 19(2), 265-275. https://recyt.fecyt.es/index.php/profesorado/article/ view/40925

Castañeda, L., Esteve, F. y Adell, J. (2018). Un Modelo Holístico de Competencia Docente para el Mundo Digital. Revista Interuniversitaria de Formación del Profesorado, 32(1), 105-116. https://recyt.fecyt.es/ index.php/RIFOP/issue/view/3377

CIFE. (2018a). Instrumento “Escala Jueces Expertos”. México. Centro Universitario CIFE.

CIFE. (2018b). Instrumento "Cuestionario de satisfacción con el instrumento". México. Centro Universitario CIFE.

Dorantes Nova, J. A., Hernández Mosqueda, J. S., Tobón Tobón, S. (2016) Juicio de expertos para la validación de un instrumento de medición del síndrome de Burnout en la docencia. Ra ximhai, 12(6), 327-346. http://revistas.unam.mx/index.php/rxm/article/view/71890 
Escobar Pérez, J., y Cuervo Martínez, A. (2008). Validez de contenido y juicio de expertos: Una aproximación a su utilización. Avances en medición, 6(1), 27-36.

García Perales, R. (2018). Diseño y construcción de un instrumento de evaluación de la competencia matemática: aplicabilidad práctica de un juicio de expertos. Ensaio Avaliação e Políticas Públicas em Educação, 26(99), 347-372. https://doi.org/10.1590/S0104-40362018002601263

García Valcalver, A., Hernández, A., Martín del Pozo, M. y Olmos, S. (2020). Validación de una rúbrica para la evaluación de trabajos fin de máster. Profesorado, 24(2). https://doi.org/10.30827/profesorado. v24i2.15151

Gómez González, J. A. (2018). Diseño y validación de un instrumento para evaluar el impacto de los proyectos formativos en educación básica en México. Atlante, 1-19. https://n9.cl/umot1

González Hernández, O. J. (2015). Validez y confiabilidad del instrumento "Percepción de comportamientos de cuidado humanizado de enfermeria PCHE za versión". Aquichan, 15(3), 381-392. http://dx.doi. org/10.5294/aqui.2015.15.3.6

Hernandez, José S; Tobón Tobón, S. (2015). Evaluación socioformativa y rendimiento académico en un programa de posgrado en línea. Paradigma, 36(1), 30-41. https://doi.org/10.37618/ PARADIGMA.1011-2251.2015.P30-41.id548

Hernández, M. G. y Cerda, M. T. (2019). Construcción y análisis de contenido de un Instrumento para evaluar la planeación didáctica en Preescolar. Atenas, 3(47). http://atenas.umcc.cu/index.php/atenas/ article/view/495

Juárez Hernández, L. G. (2018). Manual práctico de estadística básica para la investigación. ( $1^{\mathrm{a}} \mathrm{ed}$.) KResearch.

Lima Rodríguez, J. S., Lima Serrano, M., Ponce González, J. M., \& Guerra Martín, M. D. (2015). Diseño y validación de contenido de rúbricas para evaluar las competencias prácticas en estudiantes de enfermería. Revista Cubana de Educación Medica Superior 29(1), 119-133. http://www.ems.sld.cu/index. php/ems/article/view/368

Merino Soto, C., \& Livia Segovia, J. (2009). Intervalos de confianza asimétricos para el índice la validez de contenido: Un programa Visual Basic para la V de Aiken. Anales de psicología, 25(1), 169-171. https:// revistas.um.es/analesps/article/view/71631

Moscoso Loaiza, L. F. y Díaz Heredia L. P. (2018). Aspectos éticos de la investigación cualitativa con niños. Revista Latinoamericana de Bioética, 18(1), 51-67. https://doi.org/10.18359/rlbi.2955

Pacheco Hernández, P. (2015). Construcción y validación de los instrumentos para la medición de la influencia de los campos emocionales en los aprendizajes significativos. Revista Internacional de Educación y Aprendizaje, 3(1), 1-24. https://doi.org/10.37467/gka-revedu.v3.583

Parrado lozano, Y. M., Sáenz Montoya, X., Soto Lesmes, V. I., Guáqueta Parada, S. R., Amaya Rey, P., Caro Castillo, C. V., Parra Vargas, M., \& Triana Restrepo, M. C. (2015). Validez de dos instrumentos para medir la relación interpersonal de la enfermera con el paciente y su familia en la unidad de cuidado intensivo. Investigación En Enfermería: Imagen Y Desarrollo, 18(1), 115-128. https://doi.org/10.11144/ Javeriana.ie18-1.vimr

Ríos Muñoz, D., \& Herrera Araya, D. (2017). Los desafíos de la evaluación por competencias en el ámbito educativo. Scielo, 43(4) 1073-1086 https://doi.org/10.1590/S1678-4634201706164230

Robles Garrote, P., \& Rojas, M. del. C. (2015). La validación por juicio de expertos: dos investigaciones cualitativas en Lingüística aplicada. Revista Nebrija De Lingüística Aplicada a La Enseñanza De Lenguas, 9(18), 124-139. https://doi.org/10.26378/rnlael918259

Salazar Gómez, E., Tobón, S., \& Juárez Hernández, L. G. (2018). Diseño y validación de una rúbrica de evaluación de las competencias digitales desde la socioformación. Apuntes Universitarios, 8(3), 2442. https://doi.org/10.17162/au.v8i3.329

Secretaría de Educación Pública (SEP). (2017). Aprendizajes Clave para la Educación Integral. Secretaría de Educación Pública. https://cutt.ly/GWHJ4CY 
Tejada Fernández, J., \& Ruiz Bueno, C. (2015). Evaluación de competencias profesionales en educación superior: retos e implicaciones. Educación XX1, 19(1), 17-38. https://doi.org/10.5944/educxx1.12175

Tobón, S. (2017). ISSUU. Metodología de elaboración de una rubrica socioformativa. https://issuu.com/cife/docs/ metodologia_rubrica_socioformativa_

Tobón, S. (2005). Formación basada en competencias. ( $2^{\mathrm{a}}$ ed.) ECOE.

Tobón, S., Pimienta, J., Herrera, S., Gibrán, L. y Hernández, J. (2018). Validez y confiabilidad de una rúbrica para evaluar las prácticas pedagógicas en docentes de Educación Media (SOCME-10). Espacios, 39(45), 1-30.

Torres Rivera, A. D., Badillo Gaona, M., Valentin Kajatt, N. O. y Ramírez Martínez, E. T. (2014). Las competencias docentes: el desafío de la educación superior. Innovación educativa, 14(66), 129-146. https://doi.org/10.26820/reciamuc/3.(3).julio.2019.1006-1034

Valverde Berrocoso, J. \& Ciudad Gómez, A. (2014). El uso de rúbricas para la evaluación de competencias en estudiantes universitarios. Estudio sobre fiabilidad del instrumento. Revista de docencia universitaria, 12(1), 49-79. https://doi.org/10.4995/redu.2014.6415

Zambrano, R., Gil, N., Carrasco, N.y Lopera, E. (2015). Validezy confiabilidad de un instrumento de evaluación de estrategias docentes en la práctica de la enseñanza universitaria. Magister, 27(1) 26-36. https://doi. org/10.1016/j.magis.2015.06.002

\section{AUTOR}

Florentino López. Estudiante de doctorado en socioformación y sociedad del conocimiento en el Centro Universitario CIFE. Estudios de maestría en formación y práctica docente en la Universidad Pedagógica Nacional. Actualmente se desempeña como directivo escolar en la escuela primaria “Humberto Ochoa Campos”, de la ciudad de chilpancingo, Guerrero.

\section{Conflicto de intereses}

El autor declara que no existe conflicto de interés posible.

\section{Financiamiento}

No existió asistencia financiera de partes externas al presente artículo.

\section{Agradecimientos}


Anexo 1. Rúbrica para evaluar las competencias docentes en el marco de los aprendizajes clave.

\begin{tabular}{ll}
\hline Ítem & \multicolumn{1}{c}{ Niveles de dominio y descriptores } \\
\hline 1. & Autogestiona información sobre la conceptualización y organización curricular de los aprendizajes clave. \\
& Preformal: No identifica el aprendizaje clave, sólo tiene una idea básica del tema. \\
& Receptivo: Identifica información elemental de los aprendizajes clave, pero desconoce la organización curricular. \\
& Resolutivo: Conceptualiza y caracteriza los aprendizajes clave como conocimientos esenciales del currículum. \\
& Autónomo: Relaciona información fidedigna sobre los aprendizajes clave y analiza su organización curricular. \\
& Estratégico: Transfiere información fidedigna sobre los aprendizajes clave y asesora a otros sobre su definición y organización curricular, promoviendo \\
el trabajo colaborativo.
\end{tabular}

2. Planifica actividades de aprendizaje acorde con las necesidades educativas y derivadas de los aprendizajes clave.

Preformal: No planifica las actividades de aprendizaje, trabaja de acuerdo con las lecciones de los libros de texto.

Receptivo: Improvisa actividades de aprendizaje acorde a los programas de estudio.

Resolutivo: Planifica actividades de aprendizajes clave, sin considerar las necesidades educativas de los alumnos.

Autónomo: Planifica actividades de aprendizajes clave, considerando las necesidades educativas de los alumnos.

Estratégico: Crea y planifica actividades de interés para los estudiantes, partiendo de sus saberes previos, sus necesidades educativas y la gradualidad de los aprendizajes clave, para favorecer el desarrollo del talento humano.

3. Implementa estrategias de enseñanza para favorecer el logro de los aprendizajes clave en los alumnos.

Preformal: Aplica estrategias de enseñanza de forma improvisada, sin considerar su impacto en el aprendizaje.

Receptivo: Define estrategias de enseñanza tradicionalistas, sin atender los saberes previos de los alumnos.

Resolutivo: Aplica algunas estrategias de enseñanza considerando los saberes previos de los alumnos, pero sin valorar su impacto en el aprendizaje.

Autónomo: Integra estrategias de enseñanza que promueven la participación de algunos alumnos en el proceso de aprendizaje.

Estratégico: Recrea e implementa estrategias de enseñanza innovadoras que atienden los saberes previos y promueven el trabajo colaborativo en los alumnos.

4. Diseña y aplica estrategias de evaluación socioformativa para valorar el logro de los aprendizajes clave en los alumnos.

Preformal: No diseña ninguna estrategia de evaluación y evalúa de manera intuitiva el aprendizaje de los alumnos.

Receptivo: Diseña algunas estrategias de evaluación tradicionalistas para evaluar el aprendizaje.

Resolutivo: Diseña estrategias de evaluación socioformativa y sólo aplica la heteroevaluación.

Autónomo: Formula e integra estrategias de evaluación socioformativa, promoviendo la autoevaluación y coevaluación.

Estratégico: Crea y aplica estrategias de evaluación socioformativa, promoviendo la autoevaluación, coevaluación y heteroevaluación, mediante el uso de listas de cotejo, rúbricas y portafolio de evidencias.

5. Promueve el trabajo colaborativo para el logro de los aprendizajes clave.

Preformal: Conduce el proceso de enseñanza de manera verbalista e individualista.

Receptivo: Conduce la clase de manera expositiva, promoviendo la participación selectiva de los alumnos.

Resolutivo: Implementa estrategias de trabajo en equipo, sin promover la participación de todos los alumnos en el desarrollo de las actividades de aprendizaje.

Autónomo: Promueve la autorregulación del proceso de aprendizaje de los alumnos, mediante el diseño y aplicación de algunas estrategias de trabajo colaborativo.

Estratégico: Crea estrategias de trabajo colaborativo y genera ambientes de aprendizaje significativos, interactivos y democráticos con plena participación en la resolución de problemas del contexto.

6. Promueve la construcción de los aprendizajes clave en los alumnos

Preformal: Desarrolla la clase de forma expositiva y tradicionalistas, los alumnos realizan las actividades que indica el docente.

Receptivo: Controla la clase mediante actividades de lectura y copiado de textos, resolución de problemas básicos, aplicación de cuestionarios y resúmenes sin favorecer procesos metacognitivos.

Resolutivo: Organiza la clase mediante el trabajo en equipo, sin promover el rescate de saberes previos, la valoración de lo aprendido ni los procesos de retroalimentación.

Autónomo: Coordina la clase mediante el desarrollo de actividades creativas que promueven la construcción de aprendizajes clave en algunos alumnos. Estratégico: Lidera la clase mediante el diseño y desarrollo de actividades creativas, lúdicas y de interés que promueven la resolución de problemas del contexto, el trabajo colaborativo y la construcción de los aprendizajes clave en todos los alumnos.

7. A través de la intervención docente, se promueve en los alumnos la resolución de problemas del contexto.

Preformal: Se trabajan actividades de aprendizaje sin considerar la resolución de problemas del contexto.

Receptivo: Se definen algunas actividades de aprendizaje que implican la resolución de problemas descontextualizados.

Resolutivo: Implementa algunas actividades de aprendizaje que implican la resolución de problemas definidos en los libros de texto.

Autónomo: Vincula actividades de aprendizajes clave que promueven la resolución de problemas del contexto, sin realizar procesos de reflexión y socialización del conocimiento.

Estratégico: Implementa actividades de aprendizaje que sinergia el trabajo colaborativo en la resolución de problemas de contexto, generando ambientes creativos, participativos e interactivos en el aula.

8. Favorece en los alumnos el desarrollo de competencias socioemocionales.

Preformal: No se abordan las competencias socioemocionales en el trabajo cotidiano.

Receptivo: Se improvisan algunas actividades que promueven el desarrollo de competencias socioemocionales para fortalecer las relaciones interpersonales.

Resolutivo: Se implementan actividades de convivencia escolar para promover el desarrollo armónico de los educandos.

Autónomo: Se integran actividades que favorecen las competencias socioemocionales y las relaciones interpersonales entre los estudiantes.

Estratégico: Se generan ambientes de aprendizajes inclusivos y participativos, donde los alumnos autorregulan sus emociones e interactúan con el conocimiento de forma armónica y pacífica.

9. En la conducción de la práctica docente, se promueve que los alumnos construyan su proyecto ético de vida.

Preformal: Se abordan las actividades de aprendizaje, sin favorecer en los alumnos la construcción de su proyecto ético de vida.

Receptivo: Se desconocen las estrategias para promover en los alumnos la construcción de su proyecto ético de vida.

Resolutivo: Se planifican algunas estrategias que orientan a los alumnos en la definición de su proyecto ético de vida.

Autónomo: Planea metas para integrar actividades de aprendizaje que promuevan la realización personal del proyecto ético de vida.

Estratégico: Se promueve la construcción del proyecto ético de vida de los alumnos, mediante el desarrollo de actividades que favorecen la comunicación asertiva y la realización personal a partir de la práctica de valores.

10. Planifica acciones pedagógicas para que los alumnos desarrollen procesos metacognitivos.

Preformal: Aborda las clases de manera mecánica y lineal, atendiendo los contenidos de aprendizaje sin generar procesos de reflexión, ni de mejora.

Receptivo: Organiza las clases de acuerdo con el avance programático, sin considerar procesos de retroalimentación.

Resolutivo: Implementa algunas acciones orientadas a la mejora del aprendizaje de los alumnos sin promover el análisis crítico.

Autónomo: Evalúa el nivel de logro de los alumnos e integra actividades de retroalimentación y mejora del proceso de enseñanza-aprendizaje.

Estratégico: Genera espacios de reflexión, de mejora y retroalimentación del aprendizaje de los alumnos, promoviendo el desarrollo de competencias metacognitivas. 